\title{
Short-term storage of repetitions of two items'
}

\author{
HERMAN BUSCHKE 2 AND HOWARD LIM
}

STANFORD UNIVERSITY

Correct choice of the more frequent of two items presented in random sequence appears to be a function of the absolute, rather than the relative difference in number of repetitions of the items. When the absolute difference was held constant at one or five repetitions, proportions of correct choices remained constant as sequence length increased (and relative difference decreased), and was greater for an absolute difference of five than one. This suggests that such choice may depend on evaluation of the relative values of independent short-term traces for each item.

Presentation of random sequences of a few items for judgment of the relative occurrence of repetitions of each item provides a paradigm which is relevant for understanding short-term storage because it is presumably free from interference, rehearsal, and retrieval effects, and so should allow study of independent traces. The relationship of this paradigm to those which in some way require judgment of whether or not an item occurred may be viewed as that between judging whether an item occurred more than zero times $(n>k, k=0)$ in the latter case and judging whether an item occurred more than $k$ times $(n>k$, $k>0$ ) in the present case. This paradigm is of interest also because it may bear on the role of short-term storage in probability learning, perceptual sampling, and decision-making.

When a random sequence of two items (such as $\mathrm{A}$ and $B$ ) is presented at rates too rapid for overt counting (3/sec.), correct choice of the item that occurred most often is significantly better than chance, even when that item is repeated only once more than the other in a sequence of about 40 presentations (i.e., 21 and 20). That this may reflect short-term storage of independent traces for each item is suggested by the finding that when four different items (numbers $1,2,3,4)$ occur respectively 19-13-7-1, 16-12-8-4, or 13-11-9-7 times in randomly ordered sequences of 40 items, both choice of the most frequent and relative ordering of all four is better than chance and appears to be a linear function of the relative differences (Buschke, in preparation). However, increasing the number of repetitions of one item in a sequence of constant length increases both the absolute difference in number of repetitions between different items and their relative percentage differences. The present experiments were undertaken to determine whether these judgments depend on absolute or relative differences, by using a constant difference in the absolute number of repetitions of two items in random sequences of increasing length. In this case the relative difference between items decreases while the absolute difference remains constant as sequence length increases. If information about the occurrence of repetitions of two items is stored independently for each item, choice of the item more often repeated should be a function of absolute difference in number of repetitions, regardless of sequence length and relative difference. This is what was found in both experiments, which were identical except that an absolute difference of one repetition was used in Experiment 1 and an absolute difference of five in Experiment 2.

\section{Method}

Random sequences of the letters $A$ and $B$ were presented visually by an I. E. E. Binavue at $2 / \mathrm{sec}$. Twelve sequences of $31,41,51,61,71$, or 81 items were presented in random order, half of the twelve of each length having more As and half more Bs at random. In Experiment 1, the more frequent letter occurred once more often than the other (e.g., 16-15 in a sequence of 31) while in Experiment 2 it occurred five times more (e.g., 18-13). Ss responded verbally during the $4 \mathrm{sec}$, before the next sequence, giving "A" or " $B$ " as most frequent. The Ss were college students paid for their participation (16 Ss in Experiment 1 and eight Ss in Experiment 2).

\section{Resulis}

The results of both experiments are presented in Fig. 1, which shows the mean proportion correct for all Ss as a function of sequence length when the constant difference is one or five. The mean proportion correct is .595 when the difference is one and .707 when the difference is five. Both are significantly better than chance $(z=5.9, p<.001$ and $z=115.0, p<$ .001 , respectively) and proportion correct is greater with a difference of five than a difference of one for

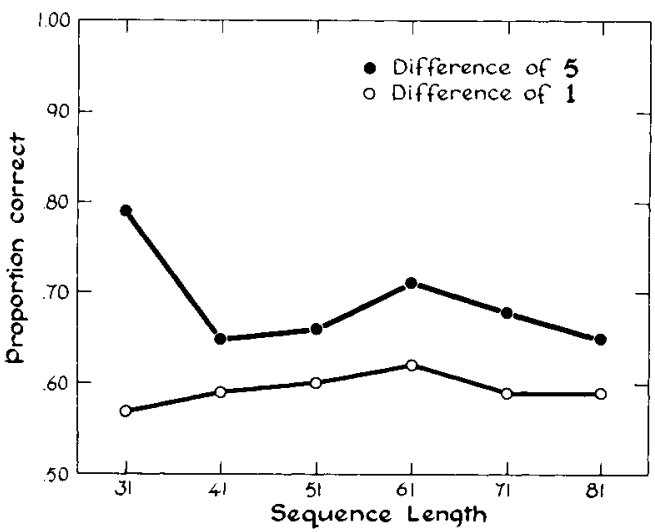

Fig. 1. Mean proportion correct with constant absolute differences of one and five repetitions as sequence length increases. 
all sequence lengths $\left(t^{2}=11.8, d f=1 / 22, p<.01\right.$; Winer, 1962). As Fig. 1 shows, there is no significant change as sequence length increases while the difference remains constant. An analysis of variance of errors by sequence length confirms the stationarity of the distribution of errors by sequence length for both differences of one and five $(F<1)$.

These results show that while proportion correct increases when the absolute difference increases and is better than chance even for a difference of only one, there is no change in proportion correct as sequence length increases for a constant absolute difference. Since proportion correct is independent of sequence length, it appears that accuracy of judgment of the more frequent of two items repeated in random sequence is a function of the absolute rather than the relative difference in the number of repetitions of the two items.

\section{Discussion}

Erlick (1962) has reported that "the percentage of correct identifications of the more frequent category when two categories are presented in a random series increases as a function of an increasing difference in the relative frequencies between the categories," and that there is a linear relationship between information transmitted and the percent increment of the more frequent category. He has found (Erlick, 1960), using the interpolated 75 percent correct identification of the more frequent event as a threshold measure, that the threshold decreases as observation time increases for both random and non-random presentation of different observation times. Overall accuracy is less for random presentation of different observation times, and the difference in performance on random and non-random presentation decreases as observation time increases. Erlick $(1960,1961)$ suggested that the input sequence may be reorganized into larger "chunks" so that frequency may be estimated by "comparison of the size and number of repetitions or clusters of the different events," that a running tally may be kept of which event is ahead, and that increased accuracy for longer observation times is due to having a larger and therefore more reliable sample on which to base judgment.

The present data show that when Ss choose which of two events repeated in random sequence occurred more often, the proportion of correct choices remains constant when the absolute difference in number of repetitions remains constant at one or five in randomly presented sequences of increasing length as the relative difference decreases. Furthermore, proportion of correct choices was significantly greater for the larger absolute difference. These findings do not seem incompatible with Erlick's reports that accuracy of judgment increases when observation time increases and relative difference remains constant (since absolute difference increases).

Following Erlick's suggestion, these results presumably might be taken to indicate that, as sequence length increases, better sampling in some sense offsets increased difficulty in discrimination due to decreased relative difference. However, this appears to assume both that judgment is based on sampling and that such samples reflect relative differences in frequency. The most parsimonious interpretation of the data would seem to be simply that judgment depends on the absolute difference in number of repetitions of the two events, which is what the present findings show and is what would be expected if there were independent short-term storage of the occurrence of repetitions of each item. This interpretation suggests that there may be an independent short-term trace (or count) for each item and that judgment is effected by comparing their relative strengths. The difficulty in judgment then reflects the difficulty of evaluating the relative strength of two traces, so that greater difference in trace strength will lead to greater probability of correct judgment.

\section{References}

Erlick, D. E. Judgments of the relative frequency of sequential binary events: Effects of frequency differences. USAF WADC tech. R'p., 1959, 59-580.

Erlick, D. E. Judgments of the relative frequency of two random sequential events: Effects of duration of observation. USAF WADD tcch. Rep., 1960, 60-673.

Erlick, D. E. Judgments of the relative frequency of a sequential series of two events. J. ixp. Psychol., 1961, 62, 105-112.

Erlick, D. E. Perception of the most frequent category of a random series as a function of the number of categories. J. cxp. Psychol., 1962, 63, 115-118.

Winer, B. J. Statistical principles in cxperimental desion. New York: McGraw-Hill, 1962. P. 32.

\section{Notes}

1. Supported by U.S.P.H. Research Grant MH-08556 to Herman Buschke from the National Institute of Mental Health.

2. Supported by U.S.P.H. Research Career Development Award K3-MH-23, 796 to Herman Buschke from the National Institute of Mental Health. 